\title{
Avances en los métodos de recuperación de oro y plata de minerales refractarios ${ }^{(\bullet)}$
}

\author{
J.R. Parga ${ }^{(*)}$ y F.R. Carrillo ${ }^{(*)}$
}

\begin{abstract}
Resumen En este artículo se analizan algunos de los avances introducidos en los procedimientos hidrometalúrgicos para la recuperación de los metales preciosos contenidos en minerales refractarios y se describen sus ventajas y aplicaciones específicas. Asimismo, se muestran dos procedimientos, la cianuración oxidante a presión en una etapa y la recuperación pirometalúrgica con plomo de metales preciosos. En el proceso de cianuración oxidante a presión, la oxidación y disolución del oro y de la plata se realizan simultáneamente en el mismo reactor, con recuperaciones de ambos superiores al $96 \%$ en 90 min. Con respecto al método de recuperación pirometalúrgica, se emplea pasta de plomo de baterías de automóviles desechadas, sin que se genere dióxido de azufre, obteniéndose también elevados valores de recuperación de los metales preciosos (>96\%).
\end{abstract}

Palabras clave: Oro. Plata. Cianuración. Oxidación a presión. Minerales refractarios. Reciclado de plomo. Desechos de plomo.

\section{Advances in the methods for gold and silver recovery from refractory ores}

\begin{abstract}
Recent research work in hydrometallurgical processes for precious metals recovery from refractory ores is analysed. Advantages and applications are discussed. Likewise, two methods, one stage direct pressure oxidation/cyanidation and pyrometallurgical recovery, are also described. In the first one, gold and silver oxidation and recovery can be carried out in the same reactor simultaneously. A higher than $96 \%$ recovery in less than 90 min can be achieved. As for the pyrometallurgical recovery method, lead paste from automotive discharged batteries is use and no sulfur dioxide is generated. A precious metals recovery higher than $96 \%$ is reached.
\end{abstract}

Keywords: Gold. Silver. Cyanidation. Pressure oxidation. Refractory ores. Lead recycling. Lead wastes.

\section{INTRODUCCIÓN}

La minería, a través de los siglos, ha formado parte de la historia y del desarrollo económico de México, siendo promotora y soporte de la expansión regional en diferentes épocas. Dentro de la industria minero-metalúrgica mexicana, la extracción de valores metálicos no férreos (oro, plata, plomo y cinc) ha tenido gran importancia, debido a la creciente demanda de metales en el mercado nacional e internacional, por ser la segunda fuente generadora de divisas para el país.

(•) Trabajo recibido el día 14 de junio de 1995.

(*) Dpto. de Metal-Mecánica. Instituto Tecnológico de Saltillo. Saltillo, México 25000 (México).
Actualmente, México es el mayor productor de plata del mundo. En cuanto al oro, afronta un déficit, pues apenas cubre la demanda interna, ya que el oro se ha obtenido típicamente como un subproducto de la producción de plata, cobre y plomo. Por ello, es necesario llevar a cabo mejoras e innovaciones en los actuales métodos de extracción de metales preciosos para aprovechar mejor los recursos naturales disponibles.

En México existen muchas menas de baja ley y otras en las que el oro y la plata se encuentran ocluidos o asociados a minerales de hierro, arsénico, manganeso y silicio, los cuales se clasifican como depósitos refractarios auríferos y argentíferos. Por lo tanto, cualquier innovación tecnológica para el mejor procesamiento de estos minerales supondría múltiples beneficios para el país. 
El procedimiento de cianuración oxidante a presión en una etapa, que se describe en el presente trabajo, es un procedimiento modificado, desarrollado experimentalmente en el Instituto Tecnológico de Saltillo y probado en la Cía. Minas de Bacís. En dicho procedimiento se combinan la oxidación a presión y la cianuración, simultáneamente, en una etapa y en un solo reactor. La cianuración y la oxidación a presión son métodos conocidos desde hace tiempo. Sin embargo, se aplican por separado y se recomiendan para diferentes minerales auríferos que los considerados en este trabajo.

\subsection{Mineralogía de los minerales refractarios}

Los minerales de oro y plata se clasifican como refractarios cuando una porción significativa de estos metales no se puede extraer eficientemente usando métodos convencionales (1-4). Los depósitos de minerales refractarios se clasifican geoquímicamente como minerales sedimentarios, los que tienen oro libre incluido, y minerales hidrotérmicos, en los que el oro está asociado con sulfuros carbonosos y compuestos de sílice (2). Ambos depósitos se consideran de difícil recuperación. El término refractario define también a los minerales de oro y plata cuya recuperación por el proceso de cianuración convencional es baja $(<80 \%)(3)$.

La causa más común de la refractabilidad es la oclusión o diseminación de finas partículas submicroscópicas de oro $(<1 \mu \mathrm{m})$ encapsuladas en los minerales de sulfuros, como la pirita $\left(\mathrm{FeS}_{2}\right)$, la arsenopirita (FeAsS), y el cuarzo $\left(\mathrm{SiO}_{2}\right)$, que son matrices insolubles y difíciles de penetrar con soluciones de cianuro en la lixiviación convencional (2-6). Otras posibles causas de la refractabilidad son las siguientes:

- Insolubilidad de minerales auríferos, como teluros, auroestibnitas y maldonitas, así como de compuestos formados durante la tostación reductora de minerales de plomo, antimonio y arsénico (3).

- Formación de capas de óxido y de compuestos de hierro, plomo, arsénico y antimonio alrededor de las partículas de oro y plata durante algunos procesos de extracción. Estas capas o películas inhiben la disolución de metales preciosos en soluciones de cianuro (3).

- Descomposición de minerales asociados, tales como pirrotita, covelita y calcocita, en compuestos complejos que consumen cianuro y que disminuyen la acción de disolución del oro por el cianuro al consumir el oxígeno durante la descomposición de dichos minerales (el proceso de cianuración requiere del oxígeno para llevar a cabo la disolución)(3).
- La presencia de minerales de carbón amorfo o materia carbonácea confiere propiedades de predepositación (preg-robbing), es decir, el carbón adsorbe el oro disuelto durante la cianuración y dificulta la extracción de este metal (3 y 5).

\subsection{Tratamiento de los minerales refractarios de oro y plata}

El método tradicional más ampliamente empleado en el tratamiento de los minerales refractarios de oro y plata es la tostación oxidante de los concentrados de flotación, que desprende el azufre, produciendo una calcina porosa que puede tratarse por cianuración. Sin embargo, esta práctica no se considera atractiva debido a la generación de $\mathrm{SO}_{2}$. $\mathrm{La}$ operación de tostación y la posterior recuperación de los metales preciosos es muy sensible a la presencia de contenidos de antimonio y de plomo en el concentrado, ya que estos elementos reducen la permeabilidad de la calcina. Además, la tostación genera gases, que contienen azufre, arsénico, antimonio y mercurio, que contaminan el medio ambiente. Estos gases pueden procesarse para la producción de ácido sulfúrico y trióxido de arsénico; sin embargo, estos procesos y su control resultan bastantes caros (4, 6 y 9$)$.

A pesar de esto, la tostación no se considera una tecnología obsoleta, pues es preferida cuando se trabaja con minerales carbonáceos. Debido a ello, este método se ha estado actualizando para disminuir sus desventajas. Ejemplos de ello son la tostación reductora y la tostación sulfurante, además de una serie de nuevas técnicas aplicadas a la tostación oxidante (6).

Por otro lado, la investigación y el desarrollo de métodos hidrometalúrgicos han atraído la atención para el tratamiento de minerales refractarios de oro y plata, como una alternativa más eficiente que la tostación (6 y 7). Este desarrollo se ha incrementado debido al alza del precio del oro, que ha llevado a la necesidad de explotar cuerpos minerales, tales como los refractarios, cuyo contenido y la mineralogía del oro no los hacían, hasta entonces, económicamente rentables, a lo que se han añadido las actuales exigencias, más estrictas, en materia de contaminación ambiental (6-8).

\section{PROCESOS HIDROMETALÚRGICOS PARA EL TRATAMIENTO DE MINERALES REFRACTARIOS DE ORO Y PLATA}

La mayoría de los desarrollos en el tratamiento de minerales de oro y plata refractarios se han dado en procesos de oxidación hidrometalúrgica, 
básicamente en tres áreas: en la oxidación hidroquímica u oxidación atmosférica en medio ácido o alcalino, en la oxidación biológica o bioquímica y, la que ha tenido mayor futuro, en la oxidación a presión con temperaturas altas, también en medio ácido o alcalino (6, 8 y 10).

\subsection{Oxidación química}

Existen varios métodos de oxidación química o hidroquímica. Uno de los principales para los minerales refractarios es la cloración, empleada más para minerales carbonáceos que para minerales piríticos, ya que estos requieren condiciones de oxidación fuertes o severas (6). El procedimiento más efectivo es emplear la cloración con una pre-oxidación con $\mathrm{Na}_{2} \mathrm{CO}_{3}$ (soda ash) para prevenir la preadsorción-depositación del oro por pasivación del material carbonáceo y, a continuación, oxidar parcialmente la matriz pirítica (11). Se han desarrollado nuevos procedimientos en la tecnología de la cloración, especialmente en el diseño de reactores de lixiviación, para elevar la efectividad del método y eliminar la etapa de preoxidación. Otro procedimiento es el del cloruro-oxígeno, que se basa en una serie de reacciones de lixiviación y precipitación:

$$
\mathrm{MS}+2 \mathrm{HCl}+1 / 2 \mathrm{O}_{(\mathrm{g})}=\mathrm{MCl}_{2}+\mathrm{S}+\mathrm{H}_{2} \mathrm{O}
$$

donde MS son sulfuros metálicos y $\mathrm{MCl}_{2}$ cloruros (de $\mathrm{Fe}, \mathrm{Cu}, \mathrm{Pb}, \mathrm{Zn}, \mathrm{Ni}$ ).

A valores de $\mathrm{pH}$ bajos (1,4-2), el $\mathrm{FeCl}_{2}$ se oxida y precipita como geothita, regenerándose el $\mathrm{HCl}$. El azufre producido se oxida a $\mathrm{H}_{2} \mathrm{SO}_{4}$ y posteriormente se trata con $\mathrm{CaCl}_{2}$ para producir $\mathrm{CaSO}_{4}$ y regenerar $\mathrm{HCl}$. La arsenopirita se oxida y produce arseniatos de hierro o de calcio o un complejo de ambos, que son insolubles. Este procedimiento fue diseñado para reducir el impacto ambiental de los residuos; sin embargo, existe el problema del medio altamente corrosivo, como es el $\mathrm{HCl}$, y su continua producción, lo cual requiere su eliminación del circuito.

También para los minerales refractarios de sulfuros (piritas y arsenopiritas) existen dos procedimientos recientes (6): el primero, NITROX, emplea $\mathrm{HNO}_{3}$ para descomponer la pirita y la arsenopirita en sulfatos:

$$
\begin{gathered}
\mathrm{FeS}_{2}+5 \mathrm{HNO}_{3}=1 / 2 \mathrm{Fe}\left(\mathrm{SO}_{4}\right)_{3}+1 / 2 \mathrm{H}_{2} \mathrm{SO}_{4}+ \\
+5 \mathrm{NO}_{(\mathrm{g})}+2 \mathrm{H}_{2} \mathrm{O}
\end{gathered}
$$

El NO producido escapa en la fase gaseosa, reacciona con el oxígeno y produce $\mathrm{NO}_{2}$, soluble en agua, regenerándose el ácido nítrico.
El proceso NITROX trabaja a $90{ }^{\circ} \mathrm{C}$, a presión atmosférica, con tiempos de retención de 1 a $2 \mathrm{~h}$, y recuperaciones superiores al $90 \%$. Además de la ventaja de trabajar en condiciones de presión atmosférica, durante la lixiviación con $\mathrm{HNO}_{3}$, el arsénico precipita como arseniato férrico y se consiguen recuperaciones de plata elevadas. Sin embargo, es preciso tratar los sulfuros del residuo oxidado y la reducción de nitratos de los efluentes, lo que añade cierto grado de complejidad y costos al proceso.

El segundo proceso, ARSENO, es una variante del anterior, ya que se lleva a cabo a 5 atm de presión de $\mathrm{O}_{2}$, a $80{ }^{\circ} \mathrm{C}$ durante $15 \mathrm{~min}$. Con este método se genera predominantemente ácido nitroso:

$$
\begin{aligned}
& 2 \mathrm{NO}_{2(\mathrm{~g})}+\mathrm{H}_{2} \mathrm{O}=\mathrm{HNO}_{2}+\mathrm{HNO}_{3} \\
& 3 \mathrm{HNO}_{2}=\mathrm{HNO}_{3}+2 \mathrm{NO}_{(\mathrm{g})}+\mathrm{H}_{2} \mathrm{O}
\end{aligned}
$$

El breve tiempo de lixiviación de este procedimiento no permite la precipitación significativa de hierro, azufre, sulfatos y arsénico presentes, que permanecen en la solución, pudiéndose recuperar la plata, ya que la jarosita no precipita.

Una variante del método ARSENO consiste en trabajar con altas temperaturas $\left(>180{ }^{\circ} \mathrm{C}\right)$ para resolver el problema de la formación de azufre, producido durante el proceso junto con la generación de arsenitas, cuya eliminación constituye un problema. En este proceso, REDOX, se añade cal para eliminar todos los sulfatos y favorecer la precipitación de arseniatos férricos (6).

\subsection{Oxidación biológica}

Existe creciente interés en la aplicación de la biolixiviación al tratamiento de menas refractarias, ya que este procedimiento ofrece un cierto grado de selectividad en la oxidación de minerales arsenopiríticos-piríticos $(4,6,10$ y 12). Una característica de este método sobre otras tecnologías de pretratamiento es que presenta diferentes velocidades de oxidación, entre las diferentes especies mineralógicas, o diferentes aspectos cristalográficos del mineral o minerales, lo que conduce a una liberación del metal de la matriz mineral sin que se requiera la oxidación completa de la misma (10).

La oxidación de los minerales sulfurosos se lleva a cabo mediante la acción de bacterias tales como la Thiobacillus ferrooxidans y la Thiobacillus thiooxidans, por combinación de dos mecanismos (6):

1) Un ataque directo microbiológico a la estructura atómica:

$$
\begin{gathered}
2 \mathrm{FeS}_{2}+2 \mathrm{H}_{2} \mathrm{O}+7 \mathrm{O}_{2}=\mathrm{FeSO}_{4}+2 \mathrm{H}_{2} \mathrm{SO}_{4} \\
2 \mathrm{FeSO}_{2}+1 / 2 \mathrm{O}_{2}+\mathrm{H}_{2} \mathrm{SO}_{4}=\mathrm{Fe}_{2}\left(\mathrm{SO}_{4}\right)_{3}+\mathrm{H}_{2} \mathrm{O}
\end{gathered}
$$


2) Un ataque indirecto por regeneración de $\mathrm{Fe}(\mathrm{II}) \mathrm{a}$ $\mathrm{Fe}(\mathrm{III})$.

$$
\begin{gathered}
\mathrm{FeS}_{2}+\mathrm{Fe}_{2}\left(\mathrm{SO}_{4}\right)_{3}=3 \mathrm{FeSO}_{4}+2 \mathrm{~S}^{\circ} \\
2 \mathrm{FeSO}_{2}+1 / 2 \mathrm{O}_{2}+\mathrm{H}_{2} \mathrm{SO}_{4}=\mathrm{Fe}_{2}\left(\mathrm{SO}_{4}\right)_{3}+1,5 \mathrm{H}_{2} \mathrm{O} \\
2 \mathrm{~S}^{\circ}+3 \mathrm{O}_{2}+2 \mathrm{H}_{2} \mathrm{O}=2 \mathrm{H}_{2} \mathrm{SO}_{4}
\end{gathered}
$$

Las bacterias precisan oxígeno y $\mathrm{CO}_{2}$ para su crecimiento y para poder oxidar los minerales. Se adaptan a valores de $\mathrm{pH}$ acusadamente ácidos (1 a 3) y su actividad es más eficiente a temperaturas comprendidas entre 30 y $40{ }^{\circ} \mathrm{C}$. La lixiviación biológica es aplicable tanto a menas (biolixiviación en montones) como a concentrados (en tanques agitados). Sin embargo, este método tiene varias limitaciones: requiere tiempos de retención muy prolongados (2-6 días), lo que lleva a altos consumos de energía para la agitación en tanques y a la necesidad de eliminar el calor producido durante la oxidación (por las reacciones exotérmicas); estas son algunas de las desventajas para la aplicación industrial de este método.

\subsection{Oxidación a presión}

La oxidación a presión se ha convertido en la mejor alternativa para la oxidación de los minerales refractarios, con muchas ventajas con relación al proceso de tostación. Tanto las menas como los concentrados pueden procesarse por oxidación a presión sin causar contaminación del medio ambiente debido a las emisiones de gases, como $\mathrm{SO}_{2}$ y arsénico $(4,6,7,9,10$ y 13).

La oxidación a presión se lleva a cabo a temperaturas entre 170 y $225^{\circ} \mathrm{C}$, presiones totales de $1.100-3.200 \mathrm{kPa}$ y presión parcial de oxígeno de 350-700 kPa, alcanzando la oxidación completa de los sulfuros y la liberación del oro y la plata en 1-3 h (9).

Comparado con el de tostación, el procedimiento de oxidación a presión tiene, como pretratamiento a la cianuración, las siguientes ventajas: elevados porcentajes de extracción de oro de los concentrados oxidados; altas recuperaciones de oro de los minerales o concentrados; es selectivo con respecto a impurezas tales como antimonio, arsénico, plomo y mercurio; ofrece una gran facilidad de manejo y de tratamiento de las impurezas $y$, por tanto, menor impacto ambiental (4, 6 y 9).

Este procedimiento se puede llevar a cabo tanto en medio alcalino como en medio ácido; sin embargo, este último ha sido el más desarrollado para aplicaciones comerciales. En el proceso alcalino (6 y 13), los contenidos de azufre y arsénico se solubilizan completamente como sales de sulfatos de sodio y arseniatos, mientras el hierro se obtiene como un residuo de $\mathrm{Fe}_{2} \mathrm{O}_{3}$. Las ventajas de esta ruta son el empleo de menores temperaturas de oxidación y la aparición de menos problemas de corrosión en las autoclaves. Al parecer, el procedimiento no ha sido atractivo debido a los altos costos del reactivo empleado, el $\mathrm{NaOH}$, y los problemas para disponer del $\mathrm{Na}_{3} \mathrm{AsO}_{4}$, un subproducto altamente contaminante (6 y 9 ).

La oxidación a presión en medio ácido es la ruta más empleada en el tratamiento de minerales refractarios. Mediante este procedimiento se consigue la oxidación completa de $\mathrm{FeS}_{2}$ y FeAsS a sulfatos y arseniatos, con una mínima formación de azufre elemental (4, 9 y 10). Este último elemento es indeseable, ya que incrementa el consumo de reactivos en la siguiente etapa de cianuración (4 y $6)$.

Las principales reacciones que ocurren en la oxidación a presión en medio ácido son:

$$
\begin{gathered}
2 \mathrm{FeS}_{2}+7 \mathrm{O}_{2}+2 \mathrm{H}_{2} \mathrm{O}=2 \mathrm{FeSO}_{4}+2 \mathrm{H}_{2} \mathrm{SO}_{4} \\
4 \mathrm{FeAsS}+11 \mathrm{O}_{2}+2 \mathrm{H}_{2} \mathrm{O}=4 \mathrm{HAsO}_{2}+4 \mathrm{FeSO}_{4} \\
\mathrm{MS}+2 \mathrm{O}_{2}=\mathrm{MSO}_{4}
\end{gathered}
$$

donde $\mathrm{M}=\mathrm{Pb}, \mathrm{Fe}, \mathrm{Zn}, \mathrm{Ni}, \mathrm{Co}$, etc.

Para evitar la formación de azufre, se precisan temperaturas elevadas $\left(180-190{ }^{\circ} \mathrm{C}\right)$. A continuación, se oxidan el ion ferroso y el ion arsénico trivalente. El consumo de ácido y las condiciones de trabajo promueven la hidrólisis y la precipitación del hierro y del arsénico como arseniatos y sulfatos férricos, hematites y jarosita (4 y 9). Para facilitar las operaciones siguientes, (neutralización de la pulpa, recuperación del oro y manejo de las colas o residuos) se prefieren los dos últimos compuestos citados (6).

Sin embargo, la recuperación de plata por el procedimiento de oxidación a presión en medio ácido es muy baja. Con este método, la plata se libera inicialmente, aunque en las condiciones de trabajo imperantes, se asocia con la jarosita, formada por hidrólisis y precipitación del ion férrico. Por lo tanto, es necesario un tratamiento posterior (con cal) para liberar la plata de la jarosita (9 y 14).

\section{OTROS MÉTODOS}

En los procedimientos descritos, se ha observado que la recuperación del oro y de la plata se lleva a cabo en dos etapas, que consisten en un pretratamiento para oxidar y liberar los valores refractarios (2-24 h) y en un tratamiento de recuperación de los mismos (comúnmente por cianuración, 24-60 h). Sin embargo, debido a estas dos etapas, aún se requieren tiempos largos (biolixiviación, cianuración) y equipo costoso (oxidación a presión). A 
continuación, se presentan dos desarrollos tecnológicos como alternativas a los procedimientos existentes: uno, la oxidación y disolución simultánea del oro y de la plata en una solución alcalina de cianuro en un autoclave ( 7 y 15) en condiciones menos drásticas $\left(80^{\circ} \mathrm{C}-550 \mathrm{kPa}\right)$. El otro, la recuperación de los metales preciosos de concentrados por un método pirometalúrgico, empleando plomo de desecho de baterías como solvente de los metales preciosos.

\subsection{Cianuración oxidante a presión en una sola etapa}

En algunos procedimientos hidrometalúrgicos se emplean presiones mayores a la atmosférica, mediante el uso de autoclaves, para acelerar las reacciones que en ellos ocurren. Tales son los casos de la oxidación a presión antes descrita, y de la cianuración a presión de minerales no refractarios de metales preciosos. Este último procedimiento ha sido estudiado por algunos investigadores (15) como una alternativa para reducir los largos tiempos que se precisan en la cianuración convencional, la cual se lleva a cabo a presión atmosférica. La ventaja del empleo de presiones elevadas en la disolución y extracción de metales preciosos se muestra en la figura 1 (15).

De acuerdo con lo anterior, se hace posible desarrollar un tratamiento para los minerales refractarios que produzca la liberación y la extracción de los metales preciosos en una sola etapa.

En este procedimiento, se verifica simultáneamente la oxidación de los sulfuros y la disolución del oro y de la plata en las siguientes condiciones de trabajo: temperatura, $80{ }^{\circ} \mathrm{C}$, y presión de oxígeno, $550 \mathrm{kPa}$. Estas condiciones permiten emplear un equipo menos sofisticado y/o costoso, por sus mínimas exigencias de energía y presión.

El procedimiento de oxidación-cianuración directa a presión (7 y 15) se lleva a cabo en un autoclave a un valor de $\mathrm{pH}$ entre 10,7 y 11,5, empleando para ello cal $(\mathrm{CaO})$ o hidróxido de sodio $(\mathrm{NaOH})$. En un medio alcalino como este, la oxidación de la pirita y la pirrotita del concentrado da lugar a la formación de sulfato ferroso, que se convierte en sulfato férrico según las siguientes reacciones (15):

$$
\begin{aligned}
& 2 \mathrm{FeS}_{2}+7 \mathrm{O}_{2}+2 \mathrm{H}_{2} \mathrm{O}=2 \mathrm{Fe}^{2+}+4 \mathrm{SO}_{4}^{2-}+4 \mathrm{H}^{+} \\
& \mathrm{FeS}_{2}+2 \mathrm{H}^{+}=\mathrm{Fe}^{2+}+\mathrm{H}_{2} \mathrm{~S}+\mathrm{S}^{\mathrm{o}} \mathrm{FeS}^{2} \mathrm{O}_{2}=\mathrm{Fe}^{2+}+\mathrm{SO}_{4}^{2-} \\
& 2 \mathrm{Fe}^{2+}+1 / 2 \mathrm{O}_{2}+2 \mathrm{H}^{+}=2 \mathrm{Fe}^{3+}+\mathrm{H}_{2} \mathrm{O}
\end{aligned}
$$

El ion férrico puede contribuir a la oxidación de la pirita, pirrotita, esfalerita y calcopirita. El azufre
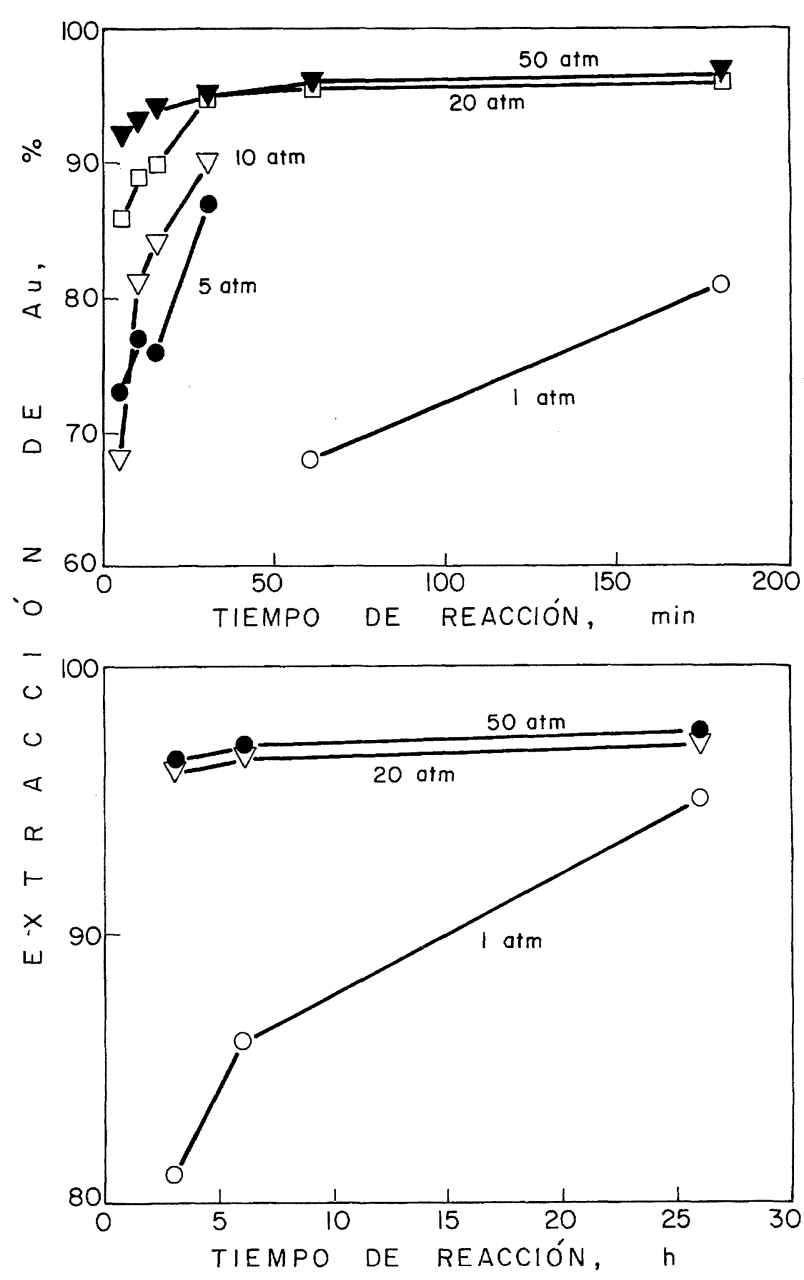

FIG. 1.- Influencia del tiempo y de la presión de oxígeno en la extracción de oro por cianuración.

FIG. 1.- Influence of time and oxygen pressure on gold extraction by cyanidation.

elemental obtenido de las reacciones anteriores se puede depositar en forma de capa porosa no protectora, sin impedir el acceso del cianuro y del oxígeno hacia el oro y la plata y así poder liberarlos. Las reacciones que se producen son:

$$
\begin{aligned}
& 4 \mathrm{Au}+8 \mathrm{CN}^{-}+\mathrm{O}_{2}+2 \mathrm{H}_{2} \mathrm{O}=4 \mathrm{Au}(\mathrm{CN})_{2}^{-}+4 \mathrm{OH}^{-} \\
& 4 \mathrm{Ag}+8 \mathrm{CN}^{-}+\mathrm{O}_{2}+2 \mathrm{H}_{2} \mathrm{O}=4 \mathrm{Ag}(\mathrm{CN})_{2}^{-}+4 \mathrm{OH}^{-}
\end{aligned}
$$

Las figuras 2 y 3 muestran los resultados cuantitativos de estas reacciones de disolución de los metales preciosos; la máxima extracción de oro y plata se alcanza transcurridos $60 \mathrm{~min}$ a $80{ }^{\circ} \mathrm{C}$. Al incrementarse ambos parámetros, se observa una disminución en la curva de extracción debido a que el cianuro se degrada por oxidación y produce cianato y, posteriormente, amonio y dióxido de carbono, tanto por su prolongada exposición al oxígeno, 


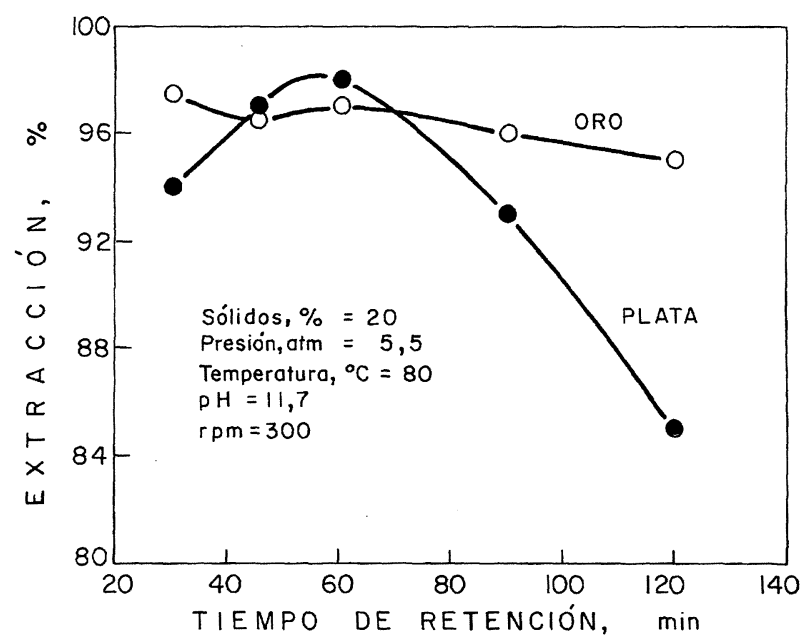

FIG. 2.- Efecto del tiempo de retención en autoclave en la extracción de oro y plata.

FIG. 2.- Effect of autoclave retention time on gold and silver extraction.

para tiempos superiores a $90 \mathrm{~min}$, como al incremento de su velocidad de oxidación a temperaturas superiores a $80{ }^{\circ} \mathrm{C}$. Los resultados experimentales muestran recuperaciones de oro y plata de aproximadamente el $96 \%$ (15).

\subsection{Procedimiento de recuperación pirometalúrgica}

El procedimiento pirometalúrgico convencional de fundición de plomo implica una serie de procesos tales la como sinterización, la reducción en hornos y el refino. De este procedimiento se obtienen oro y plata como subproductos, siendo México la principal fuente de extracción de oro. Sin embargo, esta fuente no cubre el déficit de oro que existe en el país (7 y 8). Una alternativa para resolver este problema la constituyen los minerales refractarios auríferos y argentíferos.

Por otro lado, el procedimiento pirometalúrgico debe controlarse muy bien debido a que genera muchos contaminantes (15). Además, cada vez es más patente la necesidad de recuperar y reciclar los desechos y residuos industriales, por ejemplo, las baterías de automóvil, tanto como medida de protección del medio ambiente como por los elevados costos de su manejo, transporte y eliminación, así como por la pérdida de materiales (15-17).

Debido a ésto, se propone un método para producir plomo a partir de la pasta de plomo $\left(\mathrm{PbSO}_{4}^{-}\right.$ $\mathrm{PbO}-\mathrm{Pb}$ ) de las baterías "inservibles", y recuperar metales preciosos de concentrados piríticos (15). En este procedimiento, la pasta de plomo descargada de las baterías se mezcla con el concentrado, carbonato de sodio y hierro metálico, y se funde a 950-

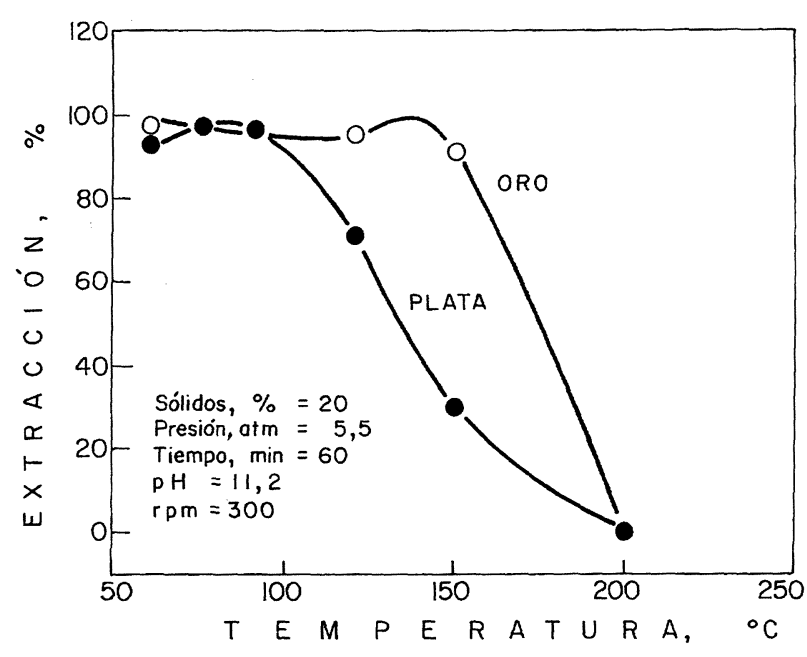

FIG. 3.- Efecto de la temperatura en la extracción de oro y plata.

FIG. 3.- Effect of temperature on gold and silver extraction.

$1.100{ }^{\circ} \mathrm{C}$, para producir un bullión de plomo y una escoria rica en hierro y sulfato de sodio. El oro y la plata se recogen en el bullión y se recuperan más tarde en procesos de refinación, tales como el proceso Parkes. En este método se añade cinc al bullión para separar los metales preciosos como una costra rica en oro y plata. La costra se funde para producir una aleación $\mathrm{Au}-\mathrm{Ag}-\mathrm{Zn}$ que pasa a un proceso de descincado (en un destilador) en el que, finalmente, se obtiene metal doré o aleación $\mathrm{Au}-\mathrm{Ag}$ (15 y 16). La figura 4 presenta un diagrama esquemático de este proceso.

En el proceso de recuperación pirometalúrgica, las emisiones de $\mathrm{SO}_{2}$ se reducen mediante adición de

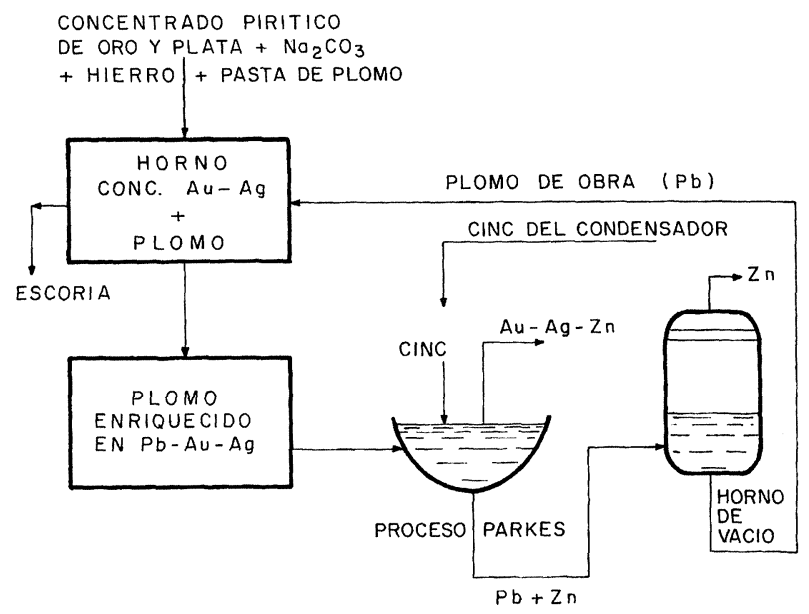

Fig. 4.- Diagrama esquemático para la recuperación de metales preciosos y plomo de concentrados piríticos.

FIG. 4.- Schematic diagram for precious metals and lead recovery from pyritic concentrates. 
carbonato de sodio, ya que el dióxido de azufre se incorpora en forma estable a la escoria de sulfato de sodio. Por otro lado, el carbonato de sodio baja el punto de fusión de la mezcla y reduce la viscosidad de la escoria, permitiendo mejorar la transferencia de especies entre la mata y la escoria y, por tanto, mejora la recuperación de los metales preciosos en el plomo.

Además, puede reaccionar con el sulfuro de plomo (del concentrado pirítico) de acuerdo con las reacciones siguientes (15):

$$
\begin{aligned}
\mathrm{PbS}+\mathrm{Na}_{2} \mathrm{CO}_{3}=\mathrm{PbO}+\mathrm{Na}_{2} \mathrm{~S}+\mathrm{SO}_{2} \\
7 \mathrm{PbO}+\mathrm{FeS}_{2}+2 \mathrm{Na}_{2} \mathrm{CO}_{3}=7 \mathrm{~Pb}+2 \mathrm{Na}_{2} \mathrm{SO}_{4}+ \\
+\mathrm{FeO}+2 \mathrm{CO}_{2}
\end{aligned}
$$

Las emisiones de partículas de plomo y de óxido de plomo también se reducen; en primer lugar, porque la capa de escoria de $\mathrm{Na}_{2} \mathrm{SO}_{4}$ formada sobre la mata evita la vaporización de plomo y óxido de plomo, y en segundo, porque este último compuesto se reduce con carbón, lo que permite recuperar, a su vez, plomo atrapado en la escoria (15):

$$
2 \mathrm{PbO}+\mathrm{C}=2 \mathrm{~Pb}+\mathrm{CO}_{2}
$$

Los resultados experimentales (15) muestran recuperaciones de hasta $97 \%$ de $\mathrm{Ag}$ y $98 \%$ de $\mathrm{Au}$, como se puede observar en las figuras 5 y 6 , trabajando con una temperatura de fusión de $1.100{ }^{\circ} \mathrm{C}$; $\mathrm{Na}_{2} \mathrm{CO}_{3}, 3,5 \%$; chatarra de hierro (como reductor), $18 \%$ en peso; carbón, $1 \%$, concentrado pirítico, 26 $\%$; pasta de plomo, 51,5\%; tiempo de fusión, de 15 a $45 \mathrm{~min}$. La variación de este último parámetro depende del tipo de horno empleado, desde un horno eléctrico de inducción, que proporciona una intensa agitación al baño y favorece la cinética de

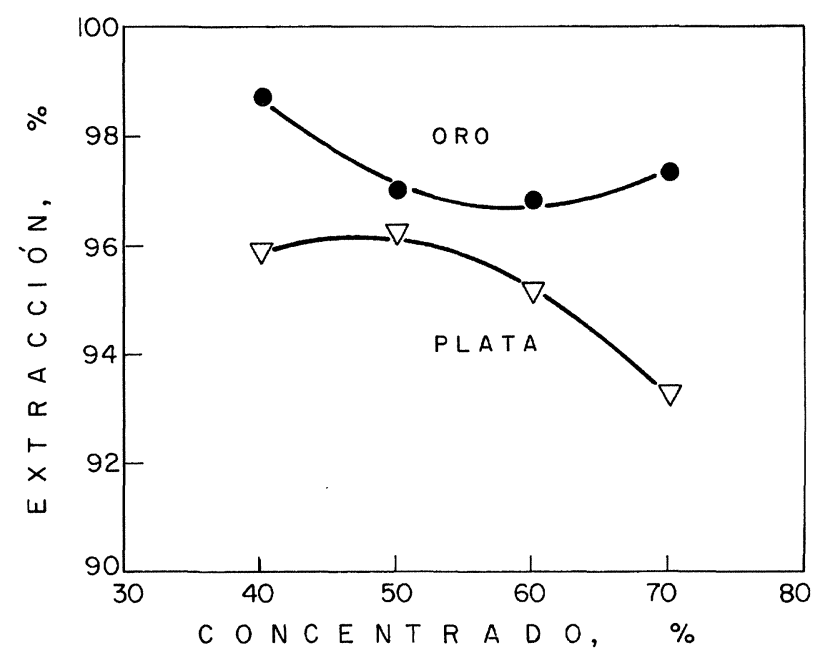

FIG. 5.- Efecto de la cantidad de concentrado pirítico.

FIG. 5.- Effect of the pyritic concentrate quantity.

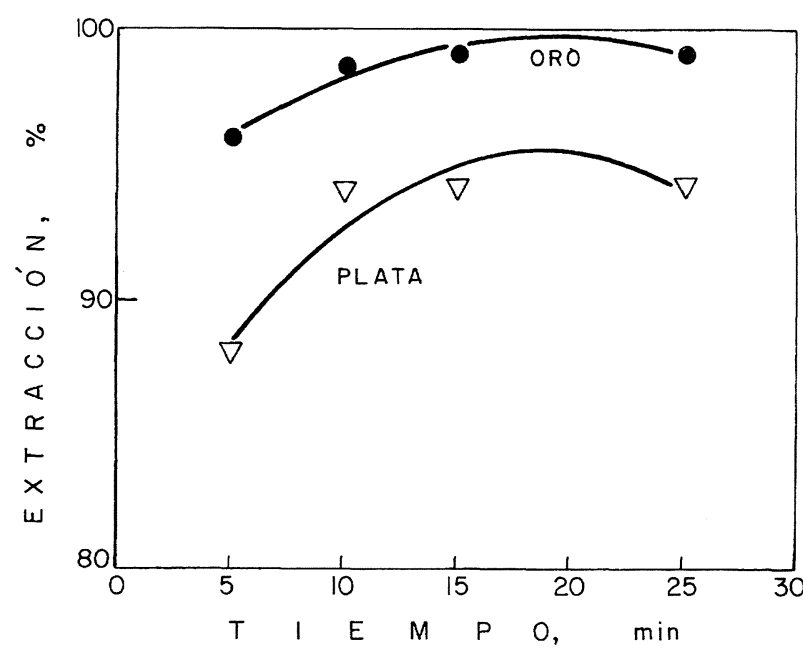

FIG. 6.- Efecto del tiempo de recuperación en la extracción de metales preciosos.

FIG. 6.- Effect of time recovery on precious metals extraction.

recuperación de valores (Figs. 7 y 8), hasta un horno de piso con gas natural, donde la agitación no es tan vigorosa, con lo que se evita la emisión de gases a la atmósfera (16).

\section{CONCLUSIONES}

El presente decenio se está caracterizando por la preocupación existente por conseguir un mejor nivel de calidad ambiental, mediante la promulgación de normas más estrictas en materia de protección del medio ambiente y poniendo mayor énfasis en el

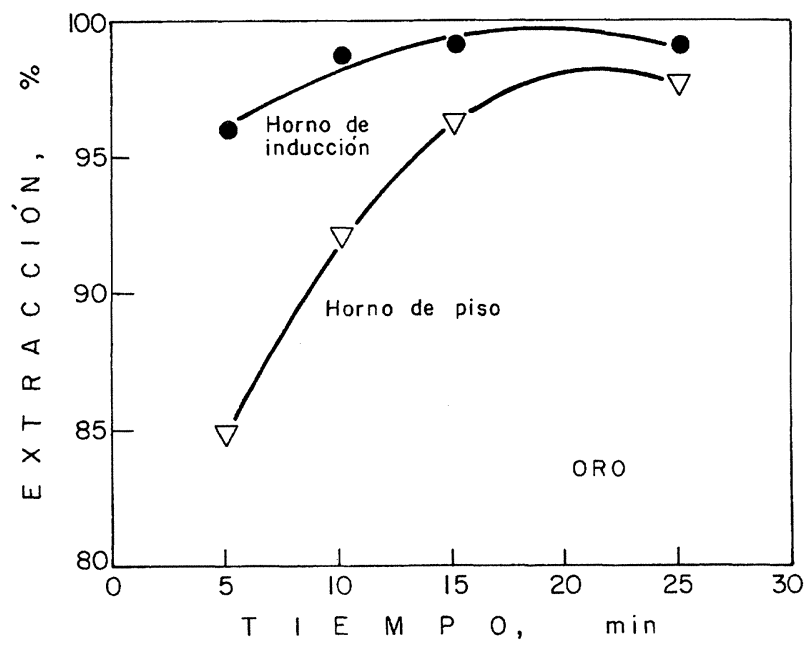

FIG. 7.- Comparación entre el horno de inducción y el de piso en la extracción de oro durante el proceso de recuperación.

FIG. 7.-Comparison of induction furnace and gasfired furnace on gold extraction in the recovery process. 


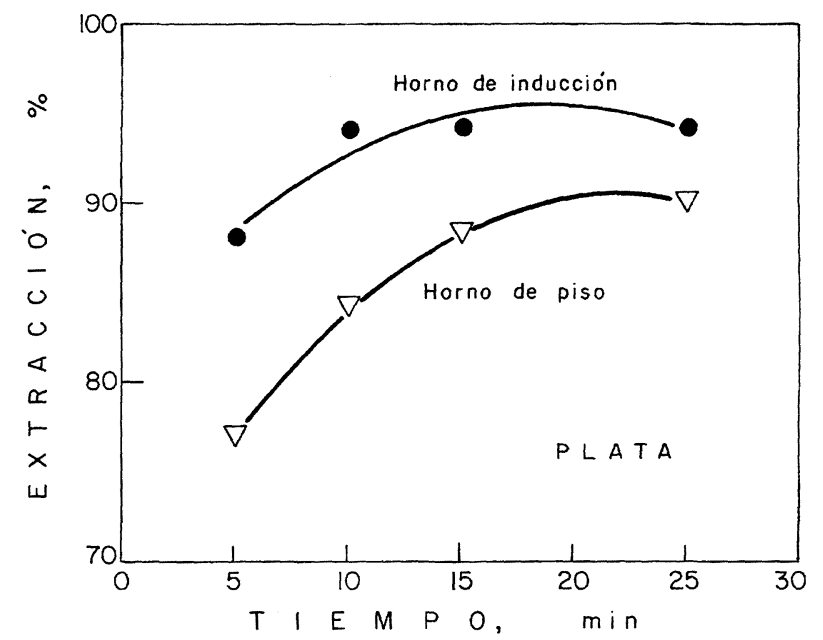

FIG. 8.- Comparación entre el horno de inducción y el de piso en la extracción de plata durante el proceso de recuperación.

FIG. 8.- Comparison on induction furnace and gas-firec furnace on silver extraction in the recovery process.

aprovechamiento de los recursos naturales, por medio de la recuperación y el reciclado de materiales y productos energéticos. Por ello, el tratamiento de minerales refractarios ha venido desarrollándose tecnológicamente, en especial en procesos hidrometalúrgicos, para satisfacer las exigencias actuales y la demanda global continua de metales preciosos.

En este trabajo se ha revisado una serie de avances tecnológicos en el campo de la recuperación de oro y plata de minerales refractarios. Esta revisión contempla los procedimientos empleados a escala comercial-industrial, como la oxidación a presión, a escala de pequeñas plantas comerciales, como la biolixiviación, y procesos que se desarrollan actualmente a escala piloto, como los métodos basados en los ácidos nítrico o clorhídrico, todos ellos con sus posibilidades, aplicaciones y limitaciones. Finalmente, se describen dos procedimientos que contribuyen de manera novedosa a ampliar las alternativas actuales en la recuperación de metales preciosos.

\section{Agradecimiento}

Los autores agradecen profundamente a la Cía. Minas de Bacís su autorización para la publicación de parte de los resultados metalúrgicos obtenidos.

\section{REFERENCIAS}

(1) White, L. Min. Eng. (N.Y.), Feb., 1990: 168-174.

(2) Chen, B. y Reddy, R.G. Roasting Characteristics of Refractory Gold Ores. Advances in Gold and Silver
Processing. Proc. Symp. at GOLDTech 4. SME-AIME. Reno, NV (EE.UU.), Sep. 1990: 201-214.

(3) Petruk, W. CIM Bulletin, Nov. 1989: 37-39.

(4) WeIR, D. Robert, Berezowsky, R.M.G.S. Refractory Gold: The Role of Pressure Oxidation. Proc. Intern. Conf. on Gold. Vol. 2: Extractive Metallurgy of Gold. SAIMM. Johannesburgo, (Rep. Sudafricana), 1986: 275-285.

(5) Marsden, J. y House, I. The Chemistry of Gold Extraction. Ed. Ellis Horwood Series. Nueva York, 1990: 60-71.

(6) Demopoulos, G.P. y Papangelakis, V.G. CIM Bulletin, Nov., 1989: 85-91.

(7) Ollervides, P.R. Desarrollo tecnológico de un proceso para recuperar metales preciosos del yacimiento de Bacis, en Durango. M.C. Tesis. Instituto Tecnológico de Saltillo, 1995.

(8) The Gold Industries of México and Central América. U.S. Bureau of Mines. Bulletin of Minerals Availability Field Office. Denver, CO (EE.UU.), 1993.

(9) Berezowski, R.M.G.S., COllins, M.J., Kerfoot,-D.G.E. y ToRrES, N. JOM, Feb., 1991: 9-15.

(10) Aguayo, S., Parga, J.R. y Sanchez, V.M. Curso "Innovaciones Minerometalúrgicas en la Recuperación de Oro y Plata". Vol. 1. Instituto Tecnológico de Saltillo, 1992: 60-74.

(11) Shoemaker, R.S. Refractory Gold Ore Processing. Advances in Gold and Silver Processing. Proc. Symp. at GOLDTech 4. SME-AIME. Reno, NV (EE.UU.), Sep. 1990: 113-118.

(12) Burbank, A., Chol, N. y Pribrey, K. Biooxidation of Refractory Gold Ores in Heaps. Advances in Gold and Silver Processing. Proc. Symp. at GOLDTech 4. SMEAIME. Reno, NV (EE.UU.), Sept. 1990: 151-159.

(13) Bhakta, P.N.H. y LeI, K.P.V. Alkaline Oxidative Pretreatment of a Low Grade Sulfidic Gold Ore. Advances in Gold and Silver Processing. Proc. Symp. at GOLDTech4. SME-AIME. Reno, NV (EE.UU.), Sept. 1990: 91-95.

(14) Scheiner, B.J., Pool, D.L., SJoberg, J.J. y Lindstrom, R.E. Extraction of Silver from Refractory Ores. Report of Investigation 7736. U.S. Bureau of Mines, 1973.

(15) Pietsch, H.P., Turke, W.M. y Rathue, G.H. Erzmetall, 36 (6), 1983: 261-265.

(16) Parga, J.R. y Mercado, H. Comparative Study of Direct Pressure Oxidation/Cyanidation and a Pirometallurgical Process for the Recovery of Precious Metals from Sulfides Concentrates. Proc. 17th Intern. Precious Metals Conf. IPMI. Newport, RI (EE.UU.), 1993: 55-67.

(17) VALDÉs, F.V. Comparación entre el horno de piso y el horno de inducción para el reciclado de plomo y recuperación de valores de oro y plata. M.C. Tesis. Instituto Tecnológico de Saltillo, 1994.

(18) Pickles, C.A. y Toguri, J.M. An Improved Pyrometallurgical Method for the Recovery of Lead. Residues and Effluents - Processing and Environmental Considerations. TMS. 1991: 313-330. 